\title{
Binding time: Evidence for integration of temporal stimulus features
}

\author{
Johanna Bogon ${ }^{1} \cdot$ Roland Thomaschke ${ }^{2}$ Gesine Dreisbach ${ }^{1}$
}

Published online: 4 May 2017

(C) The Psychonomic Society, Inc. 2017

\begin{abstract}
Several lines of evidence suggest that during processing of events, the features of these events become connected via episodic bindings. Such bindings have been demonstrated for a large number of visual and auditory stimulus features, like color and orientation, or pitch and loudness. Importantly, most visual and auditory events typically also involve temporal features, like onset time or duration. So far, however, whether temporal stimulus features are also bound into event representations has never been tested directly. The aim of the present study was to investigate possible binding between stimulus duration and other features of auditory events. In Experiment 1, participants had to respond with two keys to a low or high pitch sinus tone. Critically, the tones were presented with two different presentation durations. Sequential analysis of RT data indicated binding of stimulus duration into the event representation: at pitch repetitions, performance was better when both pitch and duration repeated, relative to when only pitch repeated and duration switched. This finding was replicated with loudness as relevant stimulus feature in Experiment 2. In sum, the results demonstrate that temporal features are bound into auditory event representations. This finding is an important advancement for binding theory in general, and raises several new questions for future research.
\end{abstract}

Johanna Bogon

johanna.bogon@psychologie.uni-regensburg.de

1 Department of Experimental Psychology, University of Regensburg, Regensburg, Germany

2 Department of Psychology, University of Freiburg, Freiburg, Germany
Keywords Feature binding $\cdot$ Partial-repetition costs $\cdot$ Binding of temporal features · Timing · Time perception

\section{Introduction}

The world consists of complex events that are characterized by diverse features. A simple event, like a traffic light turning from green to red is characterized by perceptual features, like color, form, size or location, and action features, like effector identity or intensity related to a braking action. According to one of the fundamental organizational principles of our brain, these different features of the event are processed in a distributed manner (Felleman \& Van Essen, 1991; Jeannerod, 1999; Mesulam, 1998). Several lines of evidence suggest that, during the processing of events, the perceptual and/or action features of these events become temporarily connected via episodic binding (Hommel, 1998, 2004; Kahneman, Treisman, \& Gibbs, 1992; Treisman, 1996, 1999). Such integration or binding of features has been demonstrated for response features (e.g., Hommel, 1998; Zmigrod \& Hommel, 2010), and for a large number of visual stimulus features like color, shape and orientation (e.g., Hommel, 1998; Kahneman et al., 1992), as well as for auditory stimulus features like pitch, loudness and vocal features (e.g., Bogon, Eisenbarth, Landgraf, \& Dreisbach, 2016; Moeller, Rothermund, \& Frings, 2012; Zmigrod \& Hommel, 2009). Importantly, visual and auditory events typically also involve temporal features like duration. So far, however, there is no direct evidence for the integration of temporal stimulus features.

Although binding has been demonstrated to involve almost any type of nontemporal features, it cannot automatically be inferred that binding also involves temporal features like duration. Despite time being inherent and essential to any stimulus-response event, there are potential arguments against 
binding of duration to other features. The first critical aspect is expressed by the fact that duration is an intrinsically dynamic feature, which changes over time. In contrast to an auditory stimulus feature, like pitch or loudness, that can be steadily present from the first occurrence of the stimulus until its disappearance, duration is constantly redefined. This means that, for feature binding, when the current stimulus duration is bound, the bound duration immediately becomes obsolete as the stimulus persists. Another reason preventing temporal features from becoming bound into event representations could lie in the functional role of the binding mechanism itself. Facing a dynamic environment with changing features over time, binding has been proposed to structure perception and action temporally by framing it in temporal units (Fournier \& Gallimore, 2013; Hommel \& Colzato, 2004; Pöppel, 1997; Wittmann, 2011). Here, time representations are part of the cognitive reference frame for building event representations. If temporal features themselves were integrated into event representations by binding, they can no longer serve to temporally structure perception and action. In view of this, it seems unlikely that temporal features like duration representation are integrated into event representations (cf. Thomaschke \& Dreisbach, 2015 for a similar argument). On the other hand, duration is an important feature when identifying, discriminating and processing events. Especially in the auditory domain, e.g., during music or speech processing, the duration of events plays an important role in identifying and recognizing events, and therefore its integration seems reasonable (Krumhansl, 2000; Lehiste, Olive, \& Streeter, 1976; MacGregor, Corley, \& Donaldson, 2010; Repp, 1998). Furthermore, explanations of previous findings of temporal stimulus-response compatibility effects involving stimulus duration and response duration draw on the integration of temporal features into event representations (Grosjean \& Mordkoff, 2001; Kunde, 2003; Kunde \& Stöcker, 2002).

The aim of the present study was to investigate whether temporal duration is bound in the representation of auditory events. Binding produces a characteristic sequential performance pattern, which has been used routinely to demonstrate binding effects in previous research: participants respond more slowly to partial repetitions (one feature changes, the other repeats) than to complete repetitions or complete changes of features (e.g., Hommel, 1998, 2004; Kahneman et al., 1992; Kleinsorge, 1999; Zehetleitner, Rangelov, \& Müller, 2012; Zmigrod \& Hommel, 2009, 2010). These so called partial-repetition costs are interpreted as the consequence of the automatic retrieval of previous bindings (Colzato et al., 2012; Colzato, Zmigrod, \& Hommel, 2013). Feature bindings established in one trial are retrieved in the successive trial if at least one feature is repeated. Therefore, when all features of the retrieved binding are repeated in the successive trial, no conflict occurs. There is also no conflict when none of the features of the successive trial are part of the retrieved binding (i.e., in the case of a complete change). However, when only one feature of the retrieved binding is repeated in the successive trial (partial repetition), the mismatch between the previously bound feature combination and the feature combination of the current trial causes conflict. In the present study, we used this performance pattern as an indicator for the integration of temporal stimulus features in event representations. In Experiment 1, participants had to respond with two keys to a low or high pitch sinus tone. Critically, the sinus tones were presented with two different presentation durations. If the duration is integrated in auditory event representations, sequential analyses should reveal partial-repetition costs. Repeating the duration in consecutive trials should lead to better performance if the pitch is also repeated, whereas alternation of duration should lead to better performance if the pitch is also alternated.

\section{Experiment 1}

\section{Materials and methods}

\section{Participants}

Seventeen students (age $M=23.31$ years, $S D=2.09$; one male; all right-handed) from the University of Regensburg participated for course credit. None of the participants reported any hearing impairment. One participant was excluded due to an error rate deviating 3.7 SDs from the sample mean.

\section{Apparatus and stimuli}

Participants sat in a dimly lit room facing a computer screen (19" diagonal) at a viewing distance of approximately $50 \mathrm{~cm}$. Responses were collected via the "Y" and " $\mathrm{M}$ " keys on a standard QWERTZ keyboard, positioned centrally in front of the participant. The experiment was run in E-Prime (Version 2.0, Psychology Software Tools, Sharpsburg, PA). Target stimuli were four sinewave tones with different pitch (400 Hz vs. $800 \mathrm{~Hz}$.) and duration (50 ms vs. $200 \mathrm{~ms}$ ) presented via headphones. The loudness of the tones was set at a comfortable listening level (78 dB SPL; same level for all participants), with all pitches of equal loudness.

\section{Design and procedure}

A $2 \times 2$ design was used, with the within-subject factors Pitch (repetition vs. switch) and Duration (repetition vs. switch). Participants were instructed to respond as fast and accurately as possible; to low pitch tones $(400 \mathrm{~Hz})$ with the left response key, and to high pitch tones $(800 \mathrm{~Hz})$ with the right response key. This assignment was kept constant across participants since higher tones are more associated with right positions 
and lower tones with left positions (Rusconi, Kwan, Giordano, Umiltà, \& Butterworth, 2006). Each trial started with a fixation cross of $300 \mathrm{~ms}$ duration. The target tone was then presented accompanied by a blank screen that was visible until the response was given. After an inter-trial interval of $600 \mathrm{~ms}$, the next trial started. When participants responded using the wrong button, an appropriate error message appeared for $1500 \mathrm{~ms}$. The experiment consisted of one practice block of 20 trials and three experimental blocks of 80 trials. The order of trials was randomized with the constraint that, in the experimental blocks, each factor combination (pitch sequence $\mathrm{x}$ duration sequence) appeared at least 18 times.

\section{Results and discussion}

We analyzed data from the three experimental blocks. The first trial of each block was excluded from analysis. Moreover, error trials, trials following error trials, and trials with RTs deviating more than three $S D$ s from the individual condition mean were excluded from the RT analysis (Bush, Hess, \& Wolford, 1993).

Figure 1 plots mean RTs as a function of Pitch and Duration. We conducted a 2 × 2 (Pitch: repetition vs. switch $x$ Duration: repetition vs. switch) ANOVA with repeated measures on both factors. This revealed a significant main effect of Duration, $F(1,15)=10.00, p=.006, \eta_{\mathrm{p}}{ }^{2}=.40$, indicating faster responses when the duration was repeated compared to when the duration switched ( $378 \mathrm{~ms}$ vs. $392 \mathrm{~ms}$ ). The factor Pitch was not significant $(F=1.64)$. Most importantly, there was a significant interaction Pitch $\mathrm{x}$ Duration, ${ }^{1} F(1,15)=$ $10.81, p=.005, \eta_{\mathrm{p}}^{2}=.42$. Participants responded slower in pitch repetition trials, when duration switched, compared to when duration repeated.

Figure 1 plots mean error rates as a function of Pitch and Duration. An analogous ANOVA for errors yielded a significant main effect of Pitch, $F(1,15)=4.83, p=.044, \eta_{\mathrm{p}}{ }^{2}=.24$. None of the other effects was significant (all $F_{\mathrm{S}}<.64$ ).

The Duration $x$ Pitch interaction in RT data confirmed partial-repetition costs to a certain degree: at pitch repetitions, performance was better when both pitch and duration repeated, relative to when only pitch repeated and duration switched. During pitch switches, duration did not influence responses. In order to replicate the present finding, and in order to investigate if the asymmetric performance pattern is duration specific or pitch specific, we conducted a second experiment with loudness as the relevant feature.

\footnotetext{
${ }^{1}$ In order to ascertain that this interaction is not driven by subsets of stimuli, we additionally conducted a 2 (TonePitch: low vs. high) x 2 (ToneDuration: short vs. long) x 2 (Pitch: repetition vs. switch) x 2 (Duration: repetition vs. switch) ANOVA with repeated measures on all factors. Neither the three-way interactions TonePitch $\mathrm{x}$ Pitch $\mathrm{x}$ Duration and ToneDuration $\mathrm{x}$ Pitch $\mathrm{x}$ Duration nor the four-way interaction TonePitch $\mathrm{x}$ ToneDuration $\mathrm{x}$ Pitch $\mathrm{x}$ Duration was significant (all $\left.F_{\mathrm{S}}<.11\right)$.
}

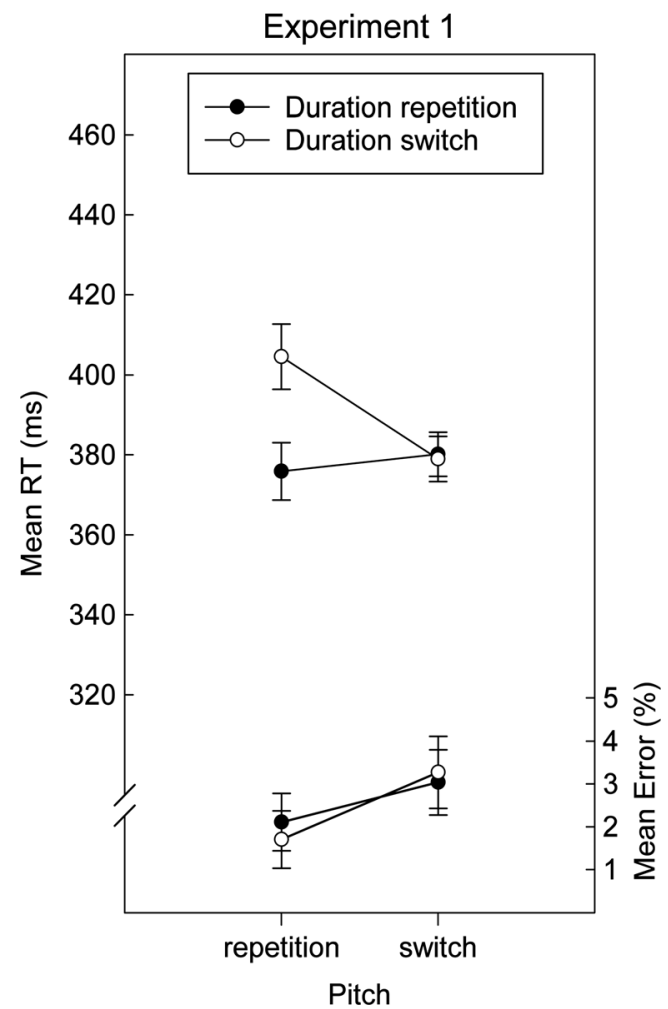

Fig. 1 Mean RTs (ms) and error rates (\%) as a function of pitch sequence (repetition vs. switch) and duration sequence (repetition vs. switch) for Experiment 1. Error bars represent inferential confidence intervals according to Tryon (2001) based on the corresponding duration repetition vs. duration switch comparison. Nonoverlap of these confidence intervals is equivalent to significance in a paired $t$-test with an alpha level of .05

\section{Experiment 2}

\section{Materials and methods}

\section{Participants}

Nineteen students (age $M=24.61$ years, $S D=2.81$; nine males; one left-handed) from the University of Regensburg participated for course credit. None of the participants reported any hearing impairment. One participant was excluded due to an error rate deviating 3.1 SDs from the sample mean.

\section{Stimuli and procedure}

The procedure of Experiment 2 mirrored that of Experiment 1, with the exception that target stimuli were four sinewave tones with different loudness (78 dB SPL vs. $60 \mathrm{~dB}$ SPL) and duration $(50 \mathrm{~ms}$ vs. $200 \mathrm{~ms})$. All participants were instructed to respond as fast and accurately as possible to soft tones with the left response key, and to loud tones with the right response key. 


\section{Results and discussion}

Preprocessing was exactly the same as in Experiment 1. Figure 2 plots mean RTs as a function of Loudness and Duration. We conducted a 2 × 2 (Loudness: repetition vs. switch x Duration: repetition vs. switch) ANOVA with repeated measures on both factors. This revealed a significant main effect of Duration, $F(1,17)=7.17, p=.016, \eta_{\mathrm{p}}{ }^{2}=.30$, indicating faster responses when the duration was repeated compared to when the duration switched (407 ms vs. $419 \mathrm{~ms})$. The factor Loudness was not significant $(F=.68)$. Most importantly, there was a significant interaction Loudness $\mathrm{x}$ Duration, ${ }^{2} F(1,17)=18.73, p<.001, \eta_{\mathrm{p}}{ }^{2}=.52$.

Figure 2 plots mean error rates as a function of Loudness and Duration. An analogous ANOVA yielded a significant main effect of Loudness $F(1,17)=5.64, p=.030, \eta_{\mathrm{p}}{ }^{2}=.25$, indicating that, overall, loudness switches were more error prone than loudness repetitions $(2.1 \%$ vs. $3.3 \%)$. None of the other effects was significant (all $F \mathrm{~s}<2.5$ ).

The RT results of Experiment 2 extended the findings of Experiment 1 . This time, the partial repetition cost pattern was more symmetric: performance was better when both loudness and duration repeated or switched, relative to when only one repeated or switched, indicating that duration is bound to other features of the auditory event.

\section{General discussion}

In two experiments, we investigated whether the duration of auditory events is integrated into coherent event representations via binding processes. Participants had to classify the pitch (Experiment 1) or the loudness (Experiment 2) of sinus tones. Irrelevant for the task, the sinus tones had two different durations. Sequential analysis of RT data indicated binding effects between stimulus duration and other stimulus features. In Experiment 1, performance was better when both pitch and duration repeated, relative to when only pitch repeated and duration switched. In Experiment 2, results revealed a more symmetric partial repetition costs pattern: performance was better when both duration and loudness switched or repeated, and was worse when only one feature switched or repeated.

Both experiments provide evidence for the integration of stimulus duration in event representations. When the taskrelevant feature repeated, performance was worse if the

\footnotetext{
${ }^{2}$ As in Experiment 1, in order to ascertain that this interaction is not driven by subsets of stimuli, we additionally conducted a 2 (ToneLoudness: low vs. high) x 2 (ToneDuration: short vs. long) x 2 (Loudness: repetition vs. switch) x 2 (Duration: repetition vs. switch) ANOVA with repeated measures on all factors. Neither the three-way interactions ToneLoudness x Loudness x Duration and ToneDuration $\mathrm{x}$ Loudness $\mathrm{x}$ Duration nor the four-way interaction ToneLoudness $\mathrm{x}$ ToneDuration $\mathrm{x}$ Loudness $\mathrm{x}$ Duration was significant (all $F$ s $<2.88)$.
}

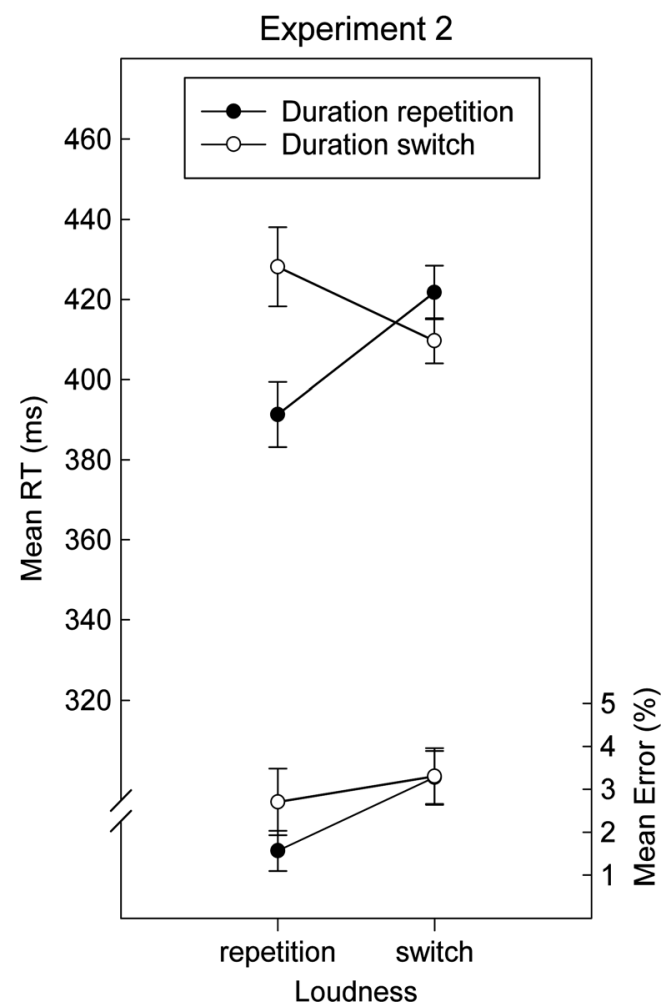

Fig. 2 Mean RTs (ms) and error rates (\%) as a function of loudness sequence (repetition vs. switch) and duration sequence (repetition vs. switch) for Experiment 2. Error bars represent inferential confidence intervals according to Tryon (2001) based on the corresponding duration repetition vs. duration switch comparison. Nonoverlap of these confidence intervals is equivalent to significance in a paired $t$-test with an alpha level of .05

duration changed compared to when the duration also repeated. That means, in both experiments, repeating the taskrelevant stimulus feature retrieved a previous binding between the task-relevant stimulus feature and the duration, resulting in costs if the previous binding mismatched the current feature combination. The capability of the task-irrelevant duration feature as retrieval cue appeared to be less solid, and seems to depend on the kind of the task-relevant feature. In Experiment 1, when the task-relevant feature switched, the repetition of the duration did not lead to performance costs. This implies that, with pitch as the relevant feature, duration failed to serve as a retrieval cue, and thus no conflict occurred even when the previous binding mismatched the actual feature combination. In Experiment 2, with loudness as the relevant feature, duration actually operated as retrieval cue: repeating duration retrieved the previous binding and caused conflict if the previous binding involved a different loudness feature than in the actual feature combination.

One might argue that our data pattern could be explained by the use of a response strategy: a change in any of the features (also duration) might evoke a tendency to change the response, strong enough to slightly delay the response execution. The use of this so-called bypass rule as a response 
strategy (Fletcher \& Rabbitt, 1978) has been discussed previously in the binding literature (e.g., Frings \& Rothermund, 2011; Giesen \& Rothermund, 2011; Mayr, Buchner, Möller, \& Hauke, 2011). Importantly, Fletcher and Rabbitt (1978) found evidence for the application of this response strategy only in highly practiced participants (five $1.5 \mathrm{~h}$ sessions prior to the experimental condition). Unpracticed participants ( $1 \mathrm{~h}$ experimental session) were found not to use this response strategy. Our experimental session lasted about $15 \mathrm{~min}$, therefore it is very unlikely that the application of a bypass rule could account for the partial repetition costs in our study. Rather, the performance patterns revealed in our experiments are the consequence of binding effects involving stimulus duration.

In our experiments, we varied duration, pitch and loudness. Previous literature reported multidimensional perceptual interactions between the perceptual dimensions involved in our study (e.g., Doughty \& Garner, 1948; Ekman, Berglund, \& Berglund, 1966). According to these perceptual interactions, the pitch or loudness of a $200 \mathrm{~ms}$ tone would be expected to be experienced differently than the pitch or loudness of a $50 \mathrm{~ms}$ tone of the same pitch or loudness. However, these interactions cannot figure as an alternative interpretation of our results. On the contrary, this compound perception would rather work statistically against our predicted binding pattern. Were a $50 \mathrm{~ms}$ and a $200 \mathrm{~ms}$ tone with objectively the same loudness or pitch to be represented so differently, there would be subjectively no partial repetitions in our design but only full repetitions and full changes. Thus, we would not find any partial repetition costs. Accordingly, we see these perceptual interactions in conceptual independence of the binding issue. Perception in one dimension biases the perception in another dimension. But still—despite their mutual interactions - the percepts of features need to be bound, to represent them as belonging to one and the same feature. Binding might be influenced by these perceptual interactions; for example, some combinations might be combined easier than others. However, these interactions would not compromise the behavioral signature of binding, i.e., partial repetition costs.

Here we have shown for the first time that temporal features can be bound to other event features. This finding is remarkable for two reasons. First, duration continually changes. Despite this constant redefinition, it is bound into event representations. Second, the flow of time is not only the content (Cole, Barnet, \& Miller, 1995; Savastano \& Miller, 1998), but also the medium, of the cognitive organization realized by binding (Fournier \& Gallimore, 2013; Hommel \& Colzato, 2004; Pöppel, 1997; Wittmann, 2011). Binding organizes feature relations in an inherently dynamic way: a certain feature combination is bound during one period of time, but unbound during another. Or, in terms of an episodic memory theory (Los, Kruijne, \& Meeter, 2014), relevant binding candidates, i.e., memory traces of feature representations, are weighted by temporal factors. We have shown that time - the actual reference frame for binding - can be bound itself, at least when it is realized as stimulus duration.

The finding that time can be bound to other features is an important advancement for binding theory in general, but it also raises several new questions. First, in the current study, duration is integrated into auditory event representations despite being irrelevant for the task. Thus, our findings may have important implications for other lines of research involving irrelevant stimulus durations (Grosjean \& Mordkoff, 2001; Kunde, 2003; Kunde \& Stöcker, 2002). Beyond that, an important question is how duration integration influences tasks that involve duration as the relevant feature (Kopec \& Brody, 2010). Second, partial-repetition costs in the present study are defined in terms of feature switches. However, in our study, a feature switch was necessarily accompanied by a response switch. Although our effects involving irrelevant stimulus duration most probably rely mainly on stimulus-stimulus integration (Zmigrod \& Hommel, 2009), it is not clear whether duration is additionally bound to the response. A number of previous binding studies explicitly dissociated between binding of features to stimulus features or to response features (Herwig \& Waszak, 2012; Hommel, 1998; Zmigrod \& Hommel, 2010). Similar designs could be employed to investigate this issue in relation to temporal features. A third important question that arises from the present findings relates to the temporal dynamics of temporal feature integration: when does the integration of duration take place, during the ongoing stimulus duration, or after its termination? Or, in other words, is temporal stimulus integration based on absolute (e.g., 200 ms; Bartolo \& Merchant, 2009; Meegan, Aslin, \& Jacobs, 2000) or relative duration representations (e.g., the longer of two stimuli; Molet \& Zentall, 2008; Pinheiro de Carvalho \& Machado, 2012; Thomaschke, Kunchulia, \& Dreisbach, 2015)? It could be an interesting question for future studies to scrutinize the temporal structure of binding temporal features.

\section{References}

Bartolo, R., \& Merchant, H. (2009). Learning and generalization of time production in humans: Rules of transfer across modalities and interval durations. Experimental Brain Research, 197(1), 91-100. doi: 10.1007/s00221-009-1895-1

Bogon, J., Eisenbarth, H., Landgraf, S., \& Dreisbach, G. (2016). Shielding voices: The modulation of binding processes between voice features and response features by task representations. The Quarterly Journal of Experimental Psychology, 70(9), 1856-1866. doi:10.1080/17470218.2016.1209686

Bush, L. K., Hess, U., \& Wolford, G. (1993). Transformations for withinsubject designs: A Monte Carlo investigation. Psychological Bulletin, 113(3), 566-579. doi:10.1037/0033-2909.113.3.566 
Cole, R. P., Barnet, R. C., \& Miller, R. R. (1995). Temporal encoding in trace conditioning. Animal Learning \& Behavior, 23(2), 144-153. doi:10.3758/BF03199929

Colzato, L. S., van Wouwe, N. C., Hommel, B., Zmigrod, S., Ridderinkhof, K. R., \& Wylie, S. A. (2012). Dopaminergic modulation of the updating of stimulus-response episodes in Parkinson's disease. Behavioural Brain Research, 228(1), 82-86. doi:10.1016/j. bbr.2011.11.034

Colzato, L. S., Zmigrod, S., \& Hommel, B. (2013). Working memory updating predicts individual differences in updating stimulus-response episodes. Visual Cognition, 21(1), 13-22. doi:10.1080/ 13506285.2013 .763883

Doughty, J. M., \& Garner, W. R. (1948). Pitch characteristics of short tones. II. Pitch as a function of tonal duration. Journal of Experimental Psychology, 38(4), 478-494. doi:10.1037/h0057850

Ekman, G., Berglund, B., \& Berglund, U. (1966). Loudness as a function of the duration of auditory stimulation. Scandinavian Journal of Psychology, 7(3), 201-208. doi:10.1111/j.1467-9450.1966. tb01354.x

Felleman, D. J., \& Van Essen, D. C. (1991). Distributed hierarchical processing in the primate cerebral cortex. Cerebral Cortex, 1(1), 1-47. doi:10.1093/cercor/1.1.1

Fletcher, B., \& Rabbitt, P. M. (1978). The changing pattern of perceptual analytic strategies and response selection with practice in a twochoice reaction time task. The Quarterly Journal of Experimental Psychology, 30(3), 417-427. doi:10.1080/00335557843000025

Fournier, L. R., \& Gallimore, J. M. (2013). What makes an event: Temporal integration of stimuli or actions? Attention, Perception, \& Psychophysics, 75(6), 1293-1305. doi:10.3758/s13414-0130461-x

Frings, C., \& Rothermund, K. (2011). To be or not to be...included in an event file: Integration and retrieval of distractors in stimulusresponse episodes is influenced by perceptual grouping. Journal of Experimental Psychology: Learning, Memory, and Cognition, 37(5), 1209-1227. doi:10.1037/a0023915

Giesen, C., \& Rothermund, K. (2011). Affective matching moderates S-R binding. Cognition \& Emotion, 25(2), 342-350. doi:10.1080/ 02699931.2010 .482765

Grosjean, M., \& Mordkoff, J. T. (2001). Temporal stimulus-response compatibility. Journal of Experimental Psychology: Human Perception and Performance, 27(4), 870-878. doi:10.1037//00961523.27.4.870

Herwig, A., \& Waszak, F. (2012). Action-effect bindings and ideomotor learning in intention and stimulus-based actions. Frontiers in Psychology, 3, 444. doi:10.3389/fpsyg.2012.00444

Hommel, B. (1998). Event Files: Evidence for automatic integration of stimulus-response episodes. Visual Cognition, 5(1-2), 183-216. doi: $10.1080 / 713756773$

Hommel, B. (2004). Event files: Feature binding in and across perception and action. Trends in Cognitive Sciences, 8(11), 494-500. doi:10. 1016/j.tics.2004.08.007

Hommel, B., \& Colzato, L. S. (2004). Visual attention and the temporal dynamics of feature integration. Visual Cognition, 11(4), 483-521. doi:10.1080/13506280344000400

Jeannerod, M. (1999). To act or not to act: Perspectives on the representation of actions. The Quarterly Journal of Experimental Psychology, 52(1), 1-29. doi:10.1080/713755803

Kahneman, D., Treisman, A., \& Gibbs, B. J. (1992). The reviewing of object files: Object-specific integration of information. Cognitive Psychology, 24(2), 175-219. doi:10.1016/0010-0285(92)90007-O

Kleinsorge, T. (1999). Response repetition benefits and costs. Acta Psychologica, 103(3), 295-310. doi:10.1016/S0001-6918(99) 00047-5

Kopec, C. D., \& Brody, C. D. (2010). Human performance on the temporal bisection task. Brain and Cognition, 74(3), 262-272. doi:10. 1016/j.bandc.2010.08.006
Krumhansl, C. L. (2000). Rhythm and pitch in music cognition. Psychological Bulletin, 126(1), 159-179. doi:10.1037/0033-2909. 126.1.159

Kunde, W. (2003). Temporal response-effect compatibility. Psychological Research, 67(3), 153-159. doi:10.1007/s00426002-0114-5

Kunde, W., \& Stöcker, C. (2002). A Simon effect for stimulus-response duration. The Quarterly Journal of Experimental Psychology, 55A(2), 581-592. doi:10.1080/0272498014300043

Lehiste, I., Olive, J. P., \& Streeter, L. A. (1976). Role of duration in disambiguating syntactically ambiguous sentences. The Journal of the Acoustical Society of America, 60(5), 1199-1202. doi:10.1121/ 1.381180

Los, S. A., Kruijne, W., \& Meeter, M. (2014). Outlines of a multiple trace theory of temporal preparation. Frontiers in Psychology, 5, 1058. doi:10.3389/fpsyg.2014.01058

MacGregor, L. J., Corley, M., \& Donaldson, D. I. (2010). Listening to the sound of silence: Disfluent silent pauses in speech have consequences for listeners. Neuropsychologia, 48(14), 3982-3992. doi: 10.1016/j.neuropsychologia.2010.09.024

Mayr, S., Buchner, A., Möller, M., \& Hauke, R. (2011). Spatial and identity negative priming in audition: Evidence of feature binding in auditory spatial memory. Attention, Perception, \& Psychophysics, 73(6), 1710-1732. doi:10.3758/s13414-011-0138-2

Meegan, D. V., Aslin, R. N., \& Jacobs, R. A. (2000). Motor timing learned without motor training. Nature Neuroscience, 3(9), 860 862. doi: $10.1038 / 78757$

Mesulam, M. (1998). From sensation to cognition. Brain, 121(6), 10131052. doi:10.1093/brain/121.6.1013

Moeller, B., Rothermund, K., \& Frings, C. (2012). Integrating the irrelevant sound. Experimental Psychology, 59(5), 258-264. doi:10. 1027/1618-3169/a000151

Molet, M., \& Zentall, T. R. (2008). Relative judgments affect assessments of stimulus duration. Psychonomic Bulletin and Review, 15(2), 431436. doi:10.3758/PBR.15.2.431

Pinheiro de Carvalho, M., \& Machado, A. (2012). Relative versus absolute stimulus control in the temporal bisection task. Journal of the Experimental Analysis of Behavior, 98(1), 23-44. doi:10.1901/jeab. 2012.98-23

Pöppel, E. (1997). A hierarchical model of temporal perception. Trends in Cognitive Sciences, 1(2), 56-61. doi:10.1016/S1364-6613(97) 01008-5

Repp, B. H. (1998). Variations on a theme by Chopin: Relations between perception and production of timing in music. Journal of Experimental Psychology: Human Perception and Performance, 24(3), 791-811. doi:10.1037//0096-1523.24.3.791

Rusconi, E., Kwan, B., Giordano, B. L., Umiltà, C., \& Butterworth, B. (2006). Spatial representation of pitch height: The SMARC effect. Cognition, 99(2), 113-129. doi:10.1016/j.cognition.2005.01.004

Savastano, H. I., \& Miller, R. R. (1998). Time as content in Pavlovian conditioning. Behavioural Processes, 44(2), 147-162. doi:10.1016/ S0376-6357(98)00046-1

Thomaschke, R., \& Dreisbach, G. (2015). The time-event correlation effect is due to temporal expectancy, not to partial transition costs. Journal of Experimental Psychology: Human Perception and Performance, 41(1), 196-218. doi:10.1037/a0038328

Thomaschke, R., Kunchulia, M., \& Dreisbach, G. (2015). Time-based event expectations employ relative, not absolute, representations of time. Psychonomic Bulletin \& Review, 22(3), 890-895. doi:10.3758/ s13423-014-0710-6

Treisman, A. (1996). The binding problem. Current Opinion in Neurobiology, 6(2), 171-178. doi:10.1016/S0959-4388(96)80070-5

Treisman, A. (1999). Solutions to the binding problem: Progress through controversy and convergence. Neuron, 24, 105-110. doi:10.1016/ S0896-6273(00)80826-0 
Tryon, W. W. (2001). Evaluating statistical difference, equivalence, and indeterminacy using inferential confidence intervals: An integrated alternative method of conducting null hypothesis statistical tests. Psychological Methods, 6(4), 371-386.

Wittmann, M. (2011). Moments in time. Frontiers in Integrative Neuroscience, 5, 66. doi:10.3389/fnint.2011.00066

Zehetleitner, M., Rangelov, D., \& Müller, H. J. (2012). Partial repetition costs persist in nonsearch compound tasks: Evidence for multiple- weighting-systems hypothesis. Attention, Perception, \& Psychophysics, 74(5), 879-890. doi:10.3758/s13414-012-0287-y

Zmigrod, S., \& Hommel, B. (2009). Auditory event files: Integrating auditory perception and action planning. Attention, Perception, \& Psychophysics, 71(2), 352-362. doi:10.3758/APP

Zmigrod, S., \& Hommel, B. (2010). Temporal dynamics of unimodal and multimodal feature binding. Attention, Perception, \& Psychophysics, 72(1), 142-152. doi:10.3758/APP 